\title{
ON THE ZEROS OF POWER SERIES WITH HADAMARD GAPS-DISTRIBUTION IN SECTORS(1)
}

BY

\section{I-LOK CHANG}

ABSTRACT. We give a sufficient condition for a power series with Hadamard gaps to assume every complex value infinitely often in every sector of the unit disk.

I. Introduction. Let

$$
f(z)=c_{0}+\sum_{k=1}^{\infty} c_{k} z^{n_{k}}
$$

be a power series convergent in $|z|<1$, with Hadamard gaps, $n_{k+1} / n_{k}>q>1$, $k \geq 1$. Given a complex number $c$, we are interested in the distribution of the zeros of $f(z)-c$. We shall discuss the problem in term of the zeros of $f$, replacing the constant term $c_{0}$ of (1) by $c_{0}-c$ if necessary.

It has been shown that

(i) $f$ has infinitely many zeros in the unit disk if $\sum_{k=0}^{k=\infty}\left|c_{k}\right|=\infty$ and $q \geq q_{0}$, where $q_{0}$ is about 100 [5].

(ii) $f$ has infinitely many zeros in any sector $\theta_{2}<\arg z<\theta_{1},|z|<1$, if $\lim \sup _{k \rightarrow \infty}\left|c_{k}\right|>0$ [2].

It remains undetermined whether $f$ has zeros in the unit disk, or perhaps in any sector, if $\sum_{k=0}^{k=\infty}\left|c_{k}\right|=\infty, \lim _{k \rightarrow \infty} c_{k}=0$, and $1<q<q_{0}$. We prove

Theorem 1. Let $f(z)=c_{0}+\sum_{k=1}^{\infty} c_{k} z^{n k}$ be a power series convergent in $|z|<1$, with

(i) $n_{k+1} / n_{k}>q>1(k \geq 1)$,

(ii) $\lim _{k \rightarrow \infty} c_{k}=0$,

(iii) $\sum_{k=0}^{\infty}\left|c_{k}\right|^{2+\epsilon}=\infty$ for some positive $\epsilon$.

Then $f$ has infinitely many zeros in any sector $\theta_{2}<\arg z<\theta_{1},|z|<1$.

II. A formula. Basic to the proof of Theorem 1 is a formula for functions

Received by the editors May 10, 1972. $30 \mathrm{~A} 70$.

AMS (MOS) subject classifications (1970). Primary 30A10; Secondary 30Q08,

Key words and phrases. Power series, coefficients, Hadamard gaps, zeros in sectors, analogue of Poisson-Jensen formula.

(1) This paper is based on part of the author's doctoral thesis written at Cornell University under Professor W.H.J. Fuchs, to whom the author wishes to express sincere appreciation. 
meromorphic in sectors. The basic idea of this formula goes back to V. P. Petrenko [3]. The following lemma can be found in [2].

Lemma 1. Suppose $f(z)$ is meromorphic in the sector $|\arg z| \leq \pi / \nu(\nu>1)$, $|z| \leq R$. Let $z=t(0<t<R)$ be a regular point of $f$ on the real axis, where $f(t) \neq 0$. For $z \neq t, R^{2} / t$, define

$$
a(z)=a(R, z, t)=\log \left|\left(R^{2}-t z\right) / R(z-t)\right|
$$

and

$$
A(R, z, t)=a(z)-a(-|z|)
$$

If we write

$$
\begin{aligned}
& I_{1}(R, t, \nu)=\int_{0}^{R}\left\{\int_{-\pi / \nu}^{\pi / \nu} \log \left|f\left(r e^{i \theta}\right)\right| d \theta\right\} \xi_{1}(R, r, t, \nu) d r \\
& I_{2}(R, t, \nu)=\int_{-\pi / \nu}^{\pi / \nu} \log \left|f\left(R e^{i \theta}\right)\right| \xi_{2}(R, \theta, t, \nu) d \theta
\end{aligned}
$$

where

$$
\begin{aligned}
& \xi_{1}(R, r, t, \nu)=\frac{\nu^{2}}{2 \pi} \frac{r^{\nu-1} t^{\nu}\left(R^{2 \nu}-t^{2 \nu}\right)\left(R^{2 \nu}-r^{2 \nu}\right)}{\left(r^{\nu}+t^{\nu}\right)^{2}\left(R^{2} \frac{1}{\left.r^{\nu} t^{2}\right)^{2}}\right.} \\
& \xi_{2}(R, \theta, t, \nu)=\frac{\nu}{\pi} \frac{R^{\nu} t^{\nu}\left(R^{\nu}-t^{\nu}\right)(1}{\left(R^{\nu}+t^{\nu}\right)\left(R^{2 \nu}+t^{2 \nu}-2 R^{\nu} t^{\nu} \cos \nu \theta\right)}
\end{aligned}
$$

then

$$
\begin{aligned}
\log |f(t)|= & I_{1}(R, t, \nu)+I_{2}(R, t, \nu)+\sum_{b_{j}} A\left(R^{\nu}, t^{\nu}, b_{j}^{\nu}\right) \\
& -\sum_{a_{i}} A\left(R^{\nu}, t^{\nu}, a_{i}^{\nu}\right)
\end{aligned}
$$

where the summation is taken over the zeros $\left\{a_{i}\right\}$ and the poles $\left\{b_{i}\right\}$ of $f$ which lie in the interior of the sector.

Without loss of generality, we may assume that $f(0)=1$ (consider $f(z) / c_{p} z^{p}$ if necessary). Suppose now that $f$ has no zero in some sector, which we may assume to be the sector $|\arg z| \leq \pi / \nu_{0},|z|<1$, where $\nu_{0}>1$. We shall show that this leads to the conclusion

$$
\limsup _{R \rightarrow 1}\left[I_{1}\left(R, 2 \nu_{0}\right)+I_{2}\left(R, 2 \nu_{0}\right)\right]=\infty
$$

whereas (2) now reduces to the contradictory result

$$
I_{1}\left(R, 2 \nu_{0}\right)+I_{2}\left(R, 2 \nu_{0}\right)=\log |f(t)| .
$$

In the next section, we derive estimates which will be used to establish (3) in $\$ I V$. 
III. Lower bounds for $|f(z)|$. Transform the domain of $f$ to the right halfplane with the change of variable $z=e^{-w}$, and write (1) as

$$
F(u)=f\left(e^{-w}\right)=c_{0}+\sum_{k=1}^{\infty} c_{k} e^{-n_{k} w}
$$

Lemma 2. There exist a subsequence $\left\{c_{k(i)}\right\}$ of the coefficients $\left\{c_{k}\right\}$ of (4) and positive constants $U_{0}(q), u_{0}(q), p_{0}(q)$ such that the derivatives of $F$ satisfy

$$
\begin{aligned}
& F^{(p)}(w)=\left(-n_{k(i)}\right)^{p} c_{k(i)} e^{-n_{k(i)} w}+R_{i}(w), \\
& \left|R_{i}(w)\right| \leq 1 / 2\left|c_{k(i)}\right| n_{k(i)}^{p} e^{-n_{k(i)} \operatorname{Re}(w)}
\end{aligned}
$$

whenever $p \geq p_{0}(q)$, and $\operatorname{Re}(w)$ is in the range

$$
u_{0}(q) / n_{k(i)}<\operatorname{Re}(w)<U_{0}(q) / n_{k(i)} .
$$

Proof. Consider the sequence $\left\{d_{k}\right\}$, where

$$
\begin{aligned}
& d_{0}=\max \left\{\left|c_{0}\right|,\left|c_{1}\right|,\left|c_{2}\right|, \ldots\right\}, \\
& d_{k}=\max \left\{1 / 2 d_{k-1},\left|c_{k}\right|,\left|c_{k+1}\right|, \ldots\right\} \quad(k \geq 1),
\end{aligned}
$$

one verifies readily that

(a) $d_{k}>0$ for all $k$,

(b) $1 / 2 \leq d_{k+1} / d_{k} \leq 1$, and also

(c) $d_{k} \geq\left|c_{k}\right|$, with equality occuring infinitely often.

If in (c), equality occured finitely often, then $d_{k+1}=1 / 2 d_{k}$ for $k \geq k_{0}$. In this case

$$
\sum_{k=k_{0}}^{\infty}\left|c_{k}\right| \leq \sum_{k=k_{0}}^{\infty} d_{k}=d_{k_{0}} \sum_{j=0}^{\infty}\left(\frac{1}{2}\right)^{j}<\infty,
$$

contradicting the assumptions that $\sum_{k=0}^{k=\infty}\left|c_{k}\right|^{2+\epsilon}=\infty$ and $\lim _{k \rightarrow \infty} c_{k}=0$.

Let $\left\{c_{k(i)}\right\}$ be the subsequence of $\left\{c_{k}\right\}$ satisfying $d_{k(i)}=\left|c_{k(i)}\right|, i=1,2, \ldots$. Differentiating $F(w) p$ times, (4) becomes

$$
F^{(p)}(w)=\sum_{k=1}^{\infty} \delta_{k} a_{k}(w)
$$

where $\delta_{k}=(-1)^{p} c_{k} / d_{k}$, and $a_{k}(w)=\left(n_{k}\right)^{p} d_{k} \exp \left(-n_{k} w\right)$. We can find, for each $k(i)$, and for sufficiently large $p$, a set of $w$ such that

$$
\begin{array}{ll}
\left|a_{k+1}(w)\right| /\left|a_{k}(w)\right|>5 & \text { for } k<k(i), \\
\left|a_{k+1}(w)\right| /\left|a_{k}(w)\right|<1 / 5 & \text { for } k \geq k(i) .
\end{array}
$$


For, (5) holds if

$$
\operatorname{Re}\left(n_{k+1} w\right)<\left(p \log t_{k}-\log 10\right) /\left(1-1 / t_{k}\right)
$$

where $t_{k}=n_{k+1} / n_{k}>q$. For sufficiently large $p$,

$$
f(t)=(p \log t-\log 10) /(1-1 / t)
$$

is a positive increasing function of $t$ in $t \geq q$. Therefore, (5) holds if $p \geq p_{0}$, and if

$$
\operatorname{Re}(w)<\left(1 / n_{k(i)}\right)(p \log q-\log 10) /(1-1 / q) .
$$

Similarly (6) holds, if

$$
\operatorname{Re}\left(n_{k} w\right)>\left(p \log t_{k}+\log 5\right) /\left(t_{k}-1\right) .
$$

The right-hand side of this inequality is bounded above by $(p \log q+\log 5) /(q-1)$, so that (6) holds if

$$
\operatorname{Re}(w)>\left(1 / n_{k(i)}\right)(p \log q+\log 5) /(q-1) .
$$

We note that if $u=(p \log q+\log 5) /(q-1)$, and $U=(p \log q-\log 10) /(1-1 / q)$, then for large $p, U / u=q(1+O(1 / p))>c>1$. Thus (5) and (6) hold simultaneously, if $p>p_{1}$, and $\operatorname{Re}(w)$ satisfies

$$
u / n_{k(i)}<\operatorname{Re}(w)<U / n_{k(i)} \text {. }
$$

If $w$ is in the range of (7), we have then

$$
\begin{aligned}
F^{(p)}(w) & =\delta_{k(i)} a_{k(i)}(w)+\sum_{k \neq k(i)} \delta_{k} a_{k}(w) \\
& =\delta_{k(i)} a_{k(i)}(w)+R_{i}(w)
\end{aligned}
$$

where $\left|\delta_{k(i)}\right|=1$, and

$$
\begin{aligned}
\left|R_{i}(w)\right| & \leq \sum_{1 \leq k<k(i)}\left|a_{k(i)}(w)\right|(5)^{k-k(i)}+\sum_{k>k(i)}\left|a_{k(i)}(w)\right|(5)^{k(i)-k} \\
& \leq 2\left|a_{k(i)}(w)\right| \sum_{j=1}^{\infty}(5)^{-j}=\frac{\left|a_{k(i)}(w)\right|}{2} .
\end{aligned}
$$

Lemma 3. Let $F(w)$ be bolomorphic in $\left|w-w_{0}\right| \leq R$. If for some $p$, $\left|F^{(p)}(w)\right| \geq m>0$ and $\sup _{\left|w-w_{0}\right| \leq R}\left|F^{(p)}(w)\right|=M$, then the image of $\left|w-w_{0}\right|$ $<R$ under $F$ covers the disk

$$
\left\{z:\left|z-F\left(w_{0}\right)\right|<K_{p} R^{p_{m}}{ }^{p+1} M-p\right\}
$$

where $K_{p}$ is a positive constant depending on $p$ only [1]. 
We infer from Lemma 2 and Lemma 3 the following

Lemma 4. If the function $f$ of Theorem 1 has no zero in the sector $|\arg z| \leq$ $\pi / \nu_{0},|z|<1$, then there exist positive constants $U_{1}, u_{1}$, and $L$, depending on $q$ only, such that $|f(z)|>L\left|c_{k(i)}\right|$ in

$$
\begin{gathered}
S_{i}: \exp \left(-U_{1} / n_{k(i)}\right)<|z|<\exp \left(-u_{1} / n_{k(i)}\right), \\
|\arg z|<\pi / \nu \quad\left(\nu=2 \nu_{0}\right) .
\end{gathered}
$$

Here $\{k(i)\}$ is the sequence defined by $\left|c_{k(i)}\right|=d_{k(i)}$.

We next estimate the size of the set of points where

$$
|f(z)|\left\{\frac{1}{2}\left(\left|c_{0}\right|^{2}+\sum_{k=1}^{\infty}\left|c_{k}\right|^{2}|z|^{2 n_{k}}\right)\right\}^{-1 / 2}
$$

is bounded away from zero. The following result is due to R. Salem and A. Zygmund. The basic idea of the proof can be found in [4]. Define

$$
A(r)=\left\{\frac{1}{2}\left(\left|c_{0}\right|^{2}+\sum_{k=1}^{\infty}\left|c_{k}\right|^{2} r^{2 n_{k}}\right)\right\}^{1 / 2} .
$$

Lemma 5. If $f$ satisfies the conditions of Theorem 1, then, in any measurable subset $E \subset[0,2 \pi]$, the linear measure

$$
m\left\{\theta \in E|| /\left(r e^{i \theta}\right) \mid A(r)^{-1} \leq y\right\}
$$

tends to $(m(E) / 2 \pi) \int_{0}^{2 \pi} \int_{0}^{y} r e^{-r^{2} / 2} d r=m(E)\left(1-e^{-y^{2 / 2}}\right)$ as $r \rightarrow 1$.

Lemma 6. For any measurable subset $E \subset[0,2 \pi]$, and any positive $\delta<1$, there is $r_{0}$ such that whenever $r \geq r_{0}$,

$$
m\left\{\theta \in E|| f\left(r e^{i \theta}\right) \mid A^{-1}(r)>\delta\right\} \geq m(E)(1-\delta) .
$$

Proof. By Lemma 5, for $r<1$,

$$
\begin{aligned}
m\left\{\theta \in E|| f\left(r e^{i \theta}\right) \mid A^{-1}(r) \leq y\right\} & =m(E)-\left\{\theta \in E|| f\left(r e^{i \theta}\right) \mid A^{-1}(r)>y\right\} \\
& \rightarrow m(E) e^{-1 / 2 y^{2}} \quad(r \rightarrow 1) .
\end{aligned}
$$

Set $y=\delta$. Since $\exp \left(-\delta^{2} ; 2\right)>1-\delta^{2} / 2>1-\delta$, (8) is proved.

IV. Lower bounds for $I_{1}(R)+I_{2}(R)$. In the following derivations, we shall use letters $K_{1}, K_{2}, K_{3}, \ldots$ for positive constants which depend on $t, t$ and $\nu$, but not on $R$.

With the notations of Lemma 1 ,

$$
I_{2}(R, t, \nu) \geq \int_{-\pi / 2 \nu}^{\pi / 2 \nu} \log ^{+}\left|f\left(\operatorname{Re}^{i \theta}\right)\right| \xi_{2} d \theta-\int_{-\pi / \nu}^{\pi / \nu} \log ^{+}\left|1 / f\left(\operatorname{Re}^{i \theta}\right)\right| \xi_{2} d \theta
$$


In the first integral of the right-hand side $\xi_{2} \geq K_{1}$ for all $R$ sufficiently close to 1. Choose $\delta$ in the interval $0<\delta<1 / 2$. By Lemma 6 , if $R \in S_{i}$ $\left(i \geq i_{0}\right), \log { }^{+}|f| \geq \log A(R)+\log \delta$ in a subset of measure $>\pi / 2 \nu$ of $(-\pi / 2 \nu, \pi / 2 \nu)$.

In the second integral $0 \leq \xi_{2} \leq K_{2}$. By Lemma $6, \log ^{+}\left|1 / f\left(\operatorname{Re}^{i \theta}\right)\right|=0$ outside a set of $\theta$ of measure $<K_{3} \delta$. In this set, by Lemma $4, \log ^{+}\left|1 / f\left(R e^{i \theta}\right)\right|<$ $-\log \left(L\left|c_{k(i)}\right|\right)$.

Therefore, for all large $i$ and $R \in S_{i}$,

$$
\begin{aligned}
I_{2} & \geq K_{4} \log A(R)+K_{4} \log \delta+K_{5} \delta \log \left|c_{k(i)}\right|-K_{6} \\
& \geq K_{7} \log A(R)+K_{4} \log \delta+K_{5} \delta \log \left|c_{k(i)}\right| .
\end{aligned}
$$

Next we find a lower bound for $I_{1}(R, t, \nu)$. From Lemma 1 ,

$$
I_{1}=2 \pi \int_{0}^{R} \xi_{1}(R, r, t, \nu)\left\{\frac{1}{2 \pi} \int_{-\pi / \nu}^{\pi / \nu} \log \left|f\left(r e^{i \theta}\right)\right| d \theta\right\} d r
$$

and we see that $\xi_{1}$ satisfies $0 \leq \xi_{1} \leq K_{8}(R-r)$. By the first fundamental theorem of Nevanlinna,

$$
\frac{1}{2 \pi} \int_{-\pi / \nu}^{\pi / \nu} \log ^{+}\left|1 / f\left(r e^{i \theta}\right)\right| d \theta \leq T(r, f)=m(r, f) .
$$

By the inequality of the arithmetic and geometric mean

$$
m(r, f) \leq K_{9} \log A(r) \leq K_{9} \log A(R) \quad(r \leq R) .
$$

Therefore, if $0<s<R$,

$$
\begin{aligned}
I_{1} \geq & 2 \pi \int_{0}^{s} \xi_{1}\left\{\frac{1}{2 \pi} \int_{-\pi / \nu}^{\pi / \nu} \log \left|f\left(r e^{i \theta}\right)\right| d \theta\right\} d r \\
& \quad-2 \pi \int_{s}^{R} \xi_{1}\left\{\frac{1}{2 \pi} \int_{-\pi / \nu}^{\pi / \nu} \log ^{+}\left|1 / f\left(r e^{i \theta}\right)\right| d \theta\right\} d r \\
\geq & D(s)-K_{10} \int_{s}^{R}(R-r) A(R) d r \geq D(s)-K_{11}(R-s)^{2} A(R) .
\end{aligned}
$$

By choosing $s$ sufficiently close to 1 , we can make

$$
K_{7}-K_{11}(R-s)^{2}>K_{7}-K_{11}(1-s)^{2}>1 / 2 K_{7}
$$

Combining (9) and (10)

$$
I_{1}+I_{2} \geq D\left(s_{0}\right)+1 / 2 K_{7} \log A(R)+K_{5} \delta \log \left|c_{k(i)}\right|+K_{4} \log \delta \quad\left(s_{0}<R\right) .
$$

Since $R \in S_{i}, A(R) \geq K_{12} \sum_{k=0}^{k(i)}\left|c_{k}\right|^{2}$, and thus

$$
I_{1}(R)+I_{2}(R) \geq K_{13}\left\{\log \left(\left|c_{k(i)}\right|^{\delta} \sum_{k=0}^{k(i)}\left|c_{k}\right|^{2}\right)+\log \delta\right\} .
$$

To show that 


$$
\lim _{R \rightarrow 1} \sup _{1}\left[I_{1}(R)+I_{2}(R)\right]=\infty
$$

it is therefore enough to show that for some $\delta$,

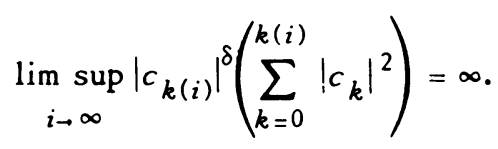

We prove first that if $0<\delta<\epsilon / 2$ where $\epsilon$ is the exponent of condition (iii) of Theorem 1, then

is infinite.

$$
W(\delta)=\lim _{p \rightarrow \infty} \sup _{p \rightarrow \infty}\left|c_{p}\right|^{\delta}\left(\sum_{k=0}^{p}\left|c_{k}\right|^{2}\right)
$$

Suppose $W(\delta)<\infty$, then for some $K>0$, and all $c_{p}$ with $\left|c_{p}\right|<1$,

$$
\left|c_{p}\right|^{2+\epsilon} \leq\left|c_{p}\right|^{2+2 \delta} \leq K\left|c_{p}\right|^{2} /\left(\sum_{k=0}^{p}\left|c_{k}\right|^{2}\right)^{2} .
$$

Summing (13) over $p$,

$$
\sum_{p=p_{0}}^{\infty}\left|c_{p}\right|^{2+\epsilon} \leq K \sum_{p=0}^{\infty}\left\{\left|c_{p}\right|^{2} /\left(\sum_{k=0}^{p}\left|c_{k}\right|^{2}\right)^{2}\right\} .
$$

The left-hand side of the inequality is infinite by assumption. The right-hand side is finite by a well-known theorem on divergent series, stating that if $a_{n} \geq 0$, and $\sum_{n=0}^{n=\infty} a_{n}=\infty$, then for any positive $\rho$,

$$
\sum_{p=0}^{\infty}\left\{a_{p} /\left(\sum_{n=0}^{p} a_{n}\right)^{1+\rho}\right\}<\infty
$$

$W(\delta)$ must therefore be infinite.

Let $S=\left|c_{p}\right|^{\delta}\left(\sum_{k=0}^{k=p}\left|c_{k}\right|^{2}\right)$. We now prove (12) by showing that for at least one of the members of the sequence $\{k(i)\}$ which are closest to $p$,

$$
\left|c_{k(i)}\right|^{\delta}\left(\sum_{k=0}^{k(i)}\left|c_{k}\right|^{2}\right)>\frac{2 S}{3} \text {. }
$$

The case $p \in\{k(i)\}$ is trivial. Suppose that $K<p$, and $K^{\prime}>p$ are the two member of $\{k(i)\}$ which are closest to $p$. If, for some $k$ in $K<k \leq p, d_{k}=\left|c_{l}\right|$ $(l>k)$, then $l \in\{k(i)\}$, and by the definition of $K$ and $K^{\prime}$, we must have $l=K^{\prime}$ and $\left|c_{p}\right|<\left|c_{K^{\prime}}\right|$, so that 


$$
\left|c_{K^{\prime}}\right|^{\delta}\left(\sum_{k=0}^{K^{\prime}}\left|c_{k}\right|^{2}\right)>\left|c_{p}\right|^{\delta}\left(\sum_{k=0}^{p}\left|c_{k}\right|^{2}\right)=S .
$$

The only other possibility is that $d_{k}=1 / 2 d_{k-1}(K<k \leq p)$ and so $\left|c_{k}\right| \leq 2^{-k+K} d_{K}$ $=2^{-k+K}\left|c_{K}\right|$,

$$
\begin{aligned}
& \sum_{k=0}^{p}\left|c_{k}\right|^{2} \leq \sum_{k=0}^{K}\left|c_{k}\right|^{2}+\left|c_{K}\right|^{2}\left(1 / 4+(1 / 4)^{2}+\cdots\right), \\
& \sum_{k=0}^{K}\left|c_{k}\right|^{2} \geq \sum_{k=0}^{p}\left|c_{k}\right|^{2}\left(1-\frac{1}{3} \frac{\left|c_{K}\right|^{2}}{\sum_{k=0}^{p}\left|c_{k}\right|^{2}}\right) \geq \frac{2}{3} \sum_{k=0}^{p}\left|c_{k}\right|^{2}
\end{aligned}
$$

if $p$ is so large that $\left|c_{K}\right|<1, \Sigma_{k=0}^{k=p}\left|c_{k}\right|^{2}>1$. We have now

$$
\left|c_{K}\right|^{\delta}\left(\sum_{k=0}^{K}\left|c_{k}\right|^{2}\right) \geq \frac{2}{3}\left|c_{p}\right|^{\delta}\left(\sum_{p=0}^{p}\left|c_{k}\right|^{2}\right)=\frac{2 S}{3} \text {. }
$$

This proves (11) and completes the proof of Theorem 1 .

\section{REFERENCES}

1. W.H.J. Fuch $\dot{\mathrm{s}}$, On the zeros of power series with Hadamard gaps, Nagoya Math. J. 29 (1967), 167-174. MR 35 \#1780.

2. Topics in Nevanlinna theory, Proc. NRL Conference on Classical Function Theory, Math. Res. Center, Naval Research Laboratory, Washington, D. C., 1970, pp. $1-32$.

3. V. P. Petrenko, Growth of meromorphic functions of finite lower order, Izv. Akad. Nauk SSSR Ser. Mat. 33 (1969), 414-454 = Math. USSR Izv. 3 (1969), 391-431. MR 40 \#4457.

4. R. Salem and A. Zygmund, On lacunary trigonometric series. I, II, Proc. Nat. Acad. Sci. U.S.A. 33 (1947), 333-338; ibid. 34 (1948), 54-62. MR 9, 181; 425.

5. M. Weiss and G. Weiss, On the Picard property of lacunary power series, Studia Math. 22 (1962/63), 221-245. MR $26 \# 2588$.

DEPARTMENT OF MATHEMATICS, AMERICAN UNIVERSITY, WASHINGTON, D. C. 20016 